\title{
Analysis on ferroresonance over-voltage of UHV converter transformer bushing tap
}

\author{
Tang Ming1, a, Ding LiJie1, b, Tang Fan1, c, Chen Gang1, d \\ ${ }^{1}$ State grid Sichuan Electric Power Research Institute, Chengdu China \\ atangming_king@163.com, bding_lijie@163.com, ${ }^{\mathrm{c}} 120610842 @ q q . c o m,{ }^{\mathrm{d}}$ gangchen08@gmail.com
}

Keywords: UHV; converter transformer; ferroresonance;

\begin{abstract}
Ferroresonance over-voltage mechanism of converter transformer bushing tap caused by charging to connection was researched, and PSCAD model was established based on actual parameters of the site for analysis. A method of increasing the damping resistor was proposed to inhibit the ferroresonance, and its influence on the accuracy of amplitude and phase was also analyzed.
\end{abstract}

\section{Introduction}

Along with the accelerated process of national strategy on west-to-east power transmission, safe and stable operation of the UHV converter station is the important grantee of sustainable transmission of clean energy.

The Xi-Zhe $\pm 800 \mathrm{kV}$ HVDC power transmission project, has the largest energy transmission capacity at present, however, is suffering from various of line fault, equipment defect and communication blocking etc. That limits HVDC from full capacity operation. Among which, ferroresonance over-voltage of converter transformer bushing tap occurred during debugging period and industrial operation. So, research on the mechanism of ferroresonance over-voltage and effectively suppress makes a significant sense on the safe and stable operation of the UHV converter.

Ferroresonance is a complex problem that puzzles power system for years [1-5], which always exists in the electrical circuit series parallel connecting inductance and capacitance [6-8]. The over-voltage and over-current can be times of rated voltage and current. Ferroresonance no only occur during operation or fault but also exist a long time after the transient process. It threatened the equipment insulation, induced over-current to burnout equipment, leaded to insulation flashover, explosion of arresters and other power grid accidents [9-11].

The converter transformer bushing tap is an important voltage monitoring node for the valve side windings, which formed a typical CVT structure with the voltage acquisition unit behind. Through the charging to connection operation, the high-end valve hall is put into operation. And this charging to connection will induce a large transient disturbance on the valve side winding through the paralleled RC of convert valve. The inductor in the voltage acquisition unit is forced to saturated region because of this large disturbance, and stable ferroresonance is formed between the converter bushing tap and voltage acquisition unit.

This paper researched the mechanism of ferroresonacne. Simulations based on electrical transient software PSCAD was held to simulated the ferroresonance fault. Various of suppress measures and their characteristics were proposed and analyzed.

\section{The Ferroresonance Over-volage Fault of the Converter Bushing Tap}

In Yibin UHV converter, during the charging to connection operation of the pole II high-end valve hall, three sets of protection system gave the same warning "Voltage transformer fault at valve side of YDA converter transformer". The voltage of each transformer valve side winding were shown in figure 1. 


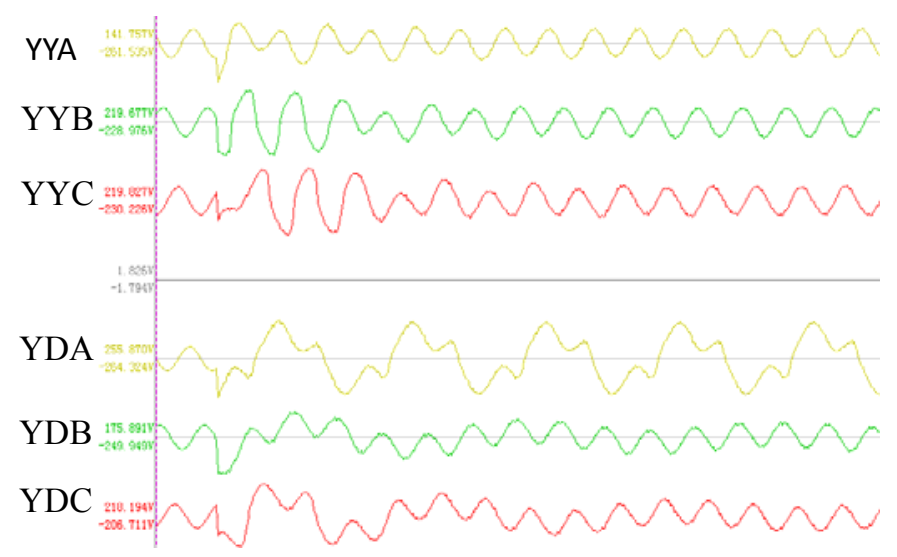

Fig.1 Waveform at bushing taps of each converter transformer in pole II

Pole I high and low-end hall, Pole II low-end was operating at rated power before the fault. The 6 convertor transformers of Pole II high-end were charged while the converter valve were blocked. The charging to connection operation is shown in figure 2.

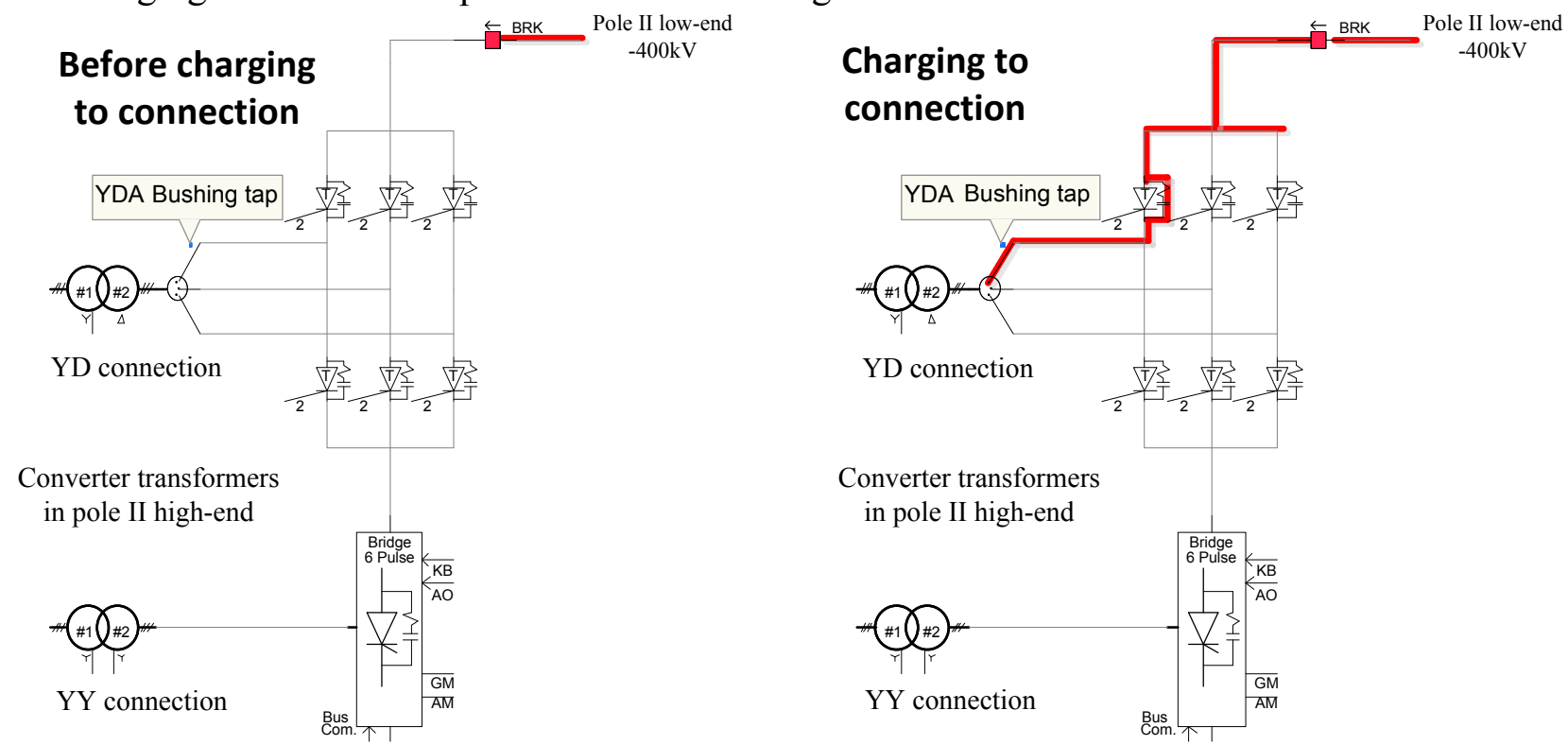

Fig.2 Demonstration of charging to connection opition

Through the charging to connection, the outline of pole II high-end valve hall connected to the $-400 \mathrm{kV}$ of pole II low-end. This operation induced a large disturbance on the valve side winding through the paralleled $\mathrm{RC}$ of convert valve. The red line in figure 2 showed the transmission direction from the disturbance to the valve side windings (other transformer was not drawn).

From the waveform record, it can be seen that the charging to connection operation induced disturbance to all the 6 converter transformers. Other 5 converter transformers' bushing tap voltage remained stable after shortly transient process while only YDA converter transformer's bushing tap formed steady ferroresonance. The spectrum analysis of the ferroresonance over-voltage is shown in figure 3 . 


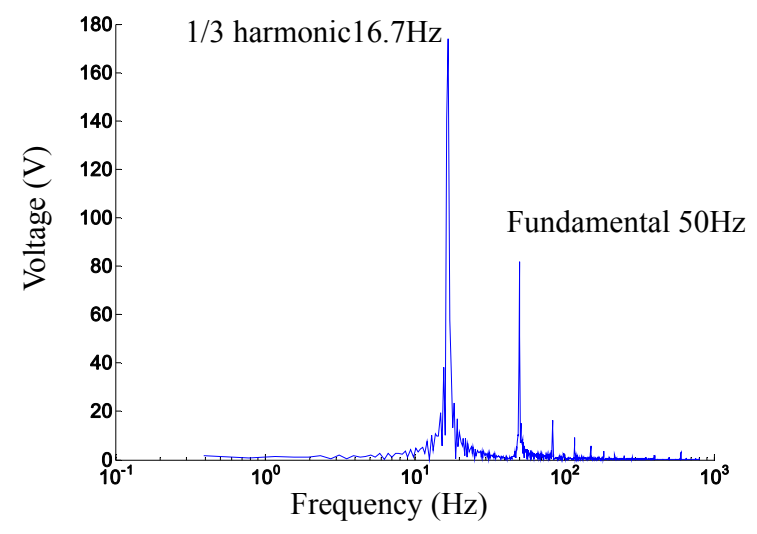

Fig.3 Frequency spectrum analysis of the tap voltage

It can be seen from figure 3 that, the amplitude of fundamental frequency component is about $82.3 \mathrm{~V}, 1 / 3$ harmonic component is $167.9 \mathrm{~V}$ which only the half of fundamental frequency. From the above analysis, it can be deduced that typical ferroresonance was occurred on the YDA converter transformer bushing tap.

Figure 4 shows the voltage divider capacitor of transformer bushing tap and its ferroresonance circuit.

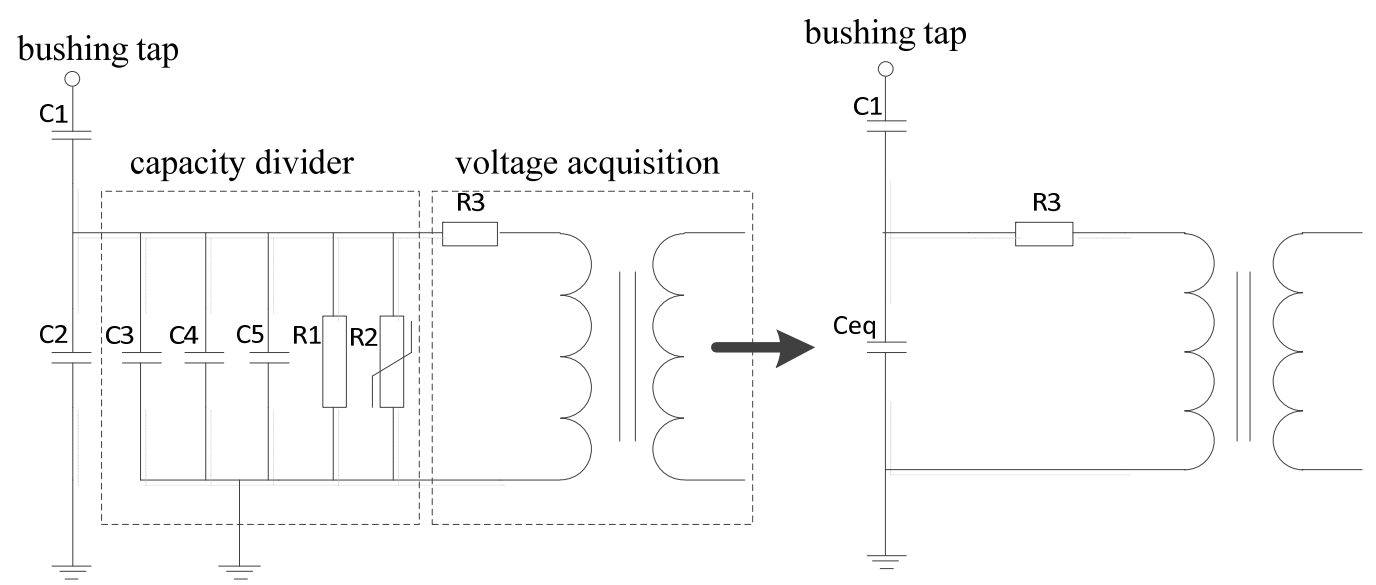

Fig.4 Ferroresonance circuit of bushing tap

The key component of the voltage acquisition unit is a small transformer with iron core. The disintegration view of the voltage acquisition unit is shown in figure 5 .

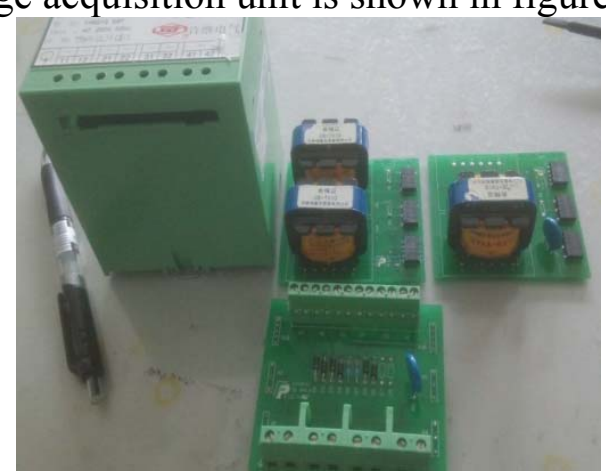

Fig.5 Object of voltage acquisition

This voltage acquisition unit is composed of 3 small PT with the same structure and parameters. The transformation ratio is 50:1. One of the detailed transformation circuit is shown in figure 6 and its main parameters are shown in table 1. 


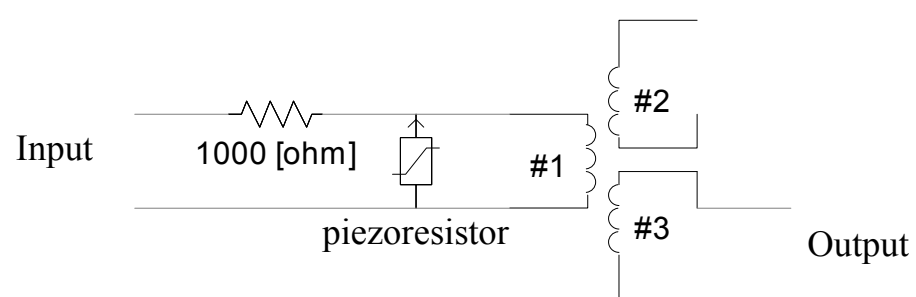

Fig. 6 Detailed circuit of voltage acquisition

The excitation characteristic curve of this acquisition unit is shown in figure 7 .

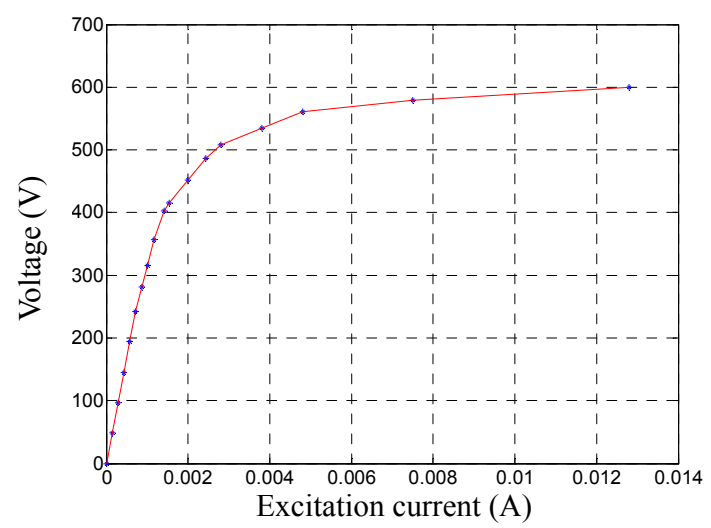

Fig.7 Excitation curve of single voltage acquisition

It should be special explained that, according to the regulations, UHV converter station adopts 3 sets protection systems and each protection system should have its own measure port independently. So, each converter transformer bushing tap is connected to 3 sets of voltage acquisition unit, and the equivalent impedance decrease to $1 / 3$.

\section{PSCAD Simulation of Ferroresonance}

Figure 8 shows the main part of ferroresonance model in PSCAD. To sufficiently simulate filed condition, parameters of each component is chosen to be consistent with real site.

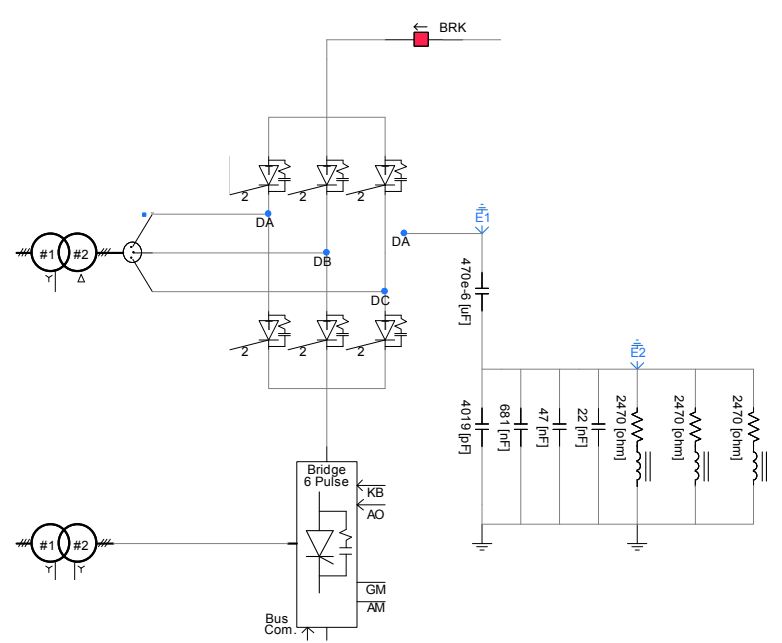

Fig.8 PSCAD model of ferroresonance in converter transformer bushing tap

A breaker and a constant voltage source are selected to simulate the operation of charging to connection. Excitation characteristic curve shown in figure 7 was adopted. Figure 9 compared the simulation result and waveform record. 


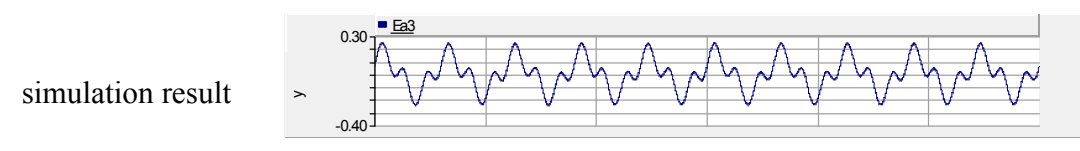

wave record

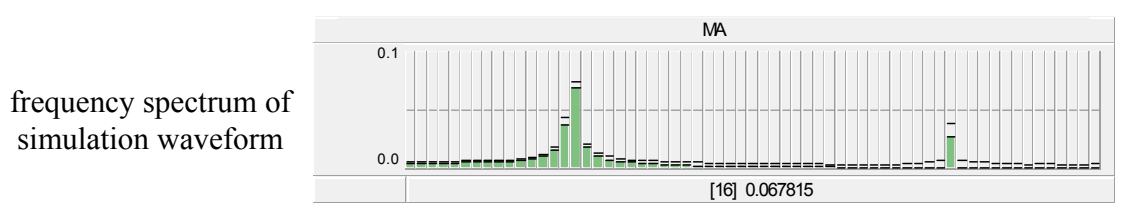

Fig.9 Simulation results and waveforms of bushing tap voltage

Simulation result showed that, voltage of converter transformer bushing tap could be dropped into steady fraction frequency resonance during the charging to connection operation. With the real site parameters, the amplitude of $1 / 3$ harmonic is nearly 2 times of the fundamental component.

\section{Suppression Measures of Ferroresonance and Analysis of Their Characteristics}

In engineering circles, the occurrence of ferroresonance depends on the $\mathrm{Xc} / \mathrm{XL}$ ratio:

$\mathrm{Xc} / \mathrm{XL}=0.01-0.07$, fraction frequency resonance;

$\mathrm{Xc} / \mathrm{XL}=0.07-0.55$, fundamental frequency resonance;

$\mathrm{Xc} / \mathrm{XL}=0.55-2.8$, high frequency resonance;

$\mathrm{Xc} / \mathrm{XL}<0.01$ or $\mathrm{Xc} / \mathrm{XL}>2.8$, hard to ferroresonance;

So, the occurrence probability of ferroresonance could be decreased by adjusting $\mathrm{Xc} / \mathrm{XL}$ ratio.

(1)Decrease Xc

Increasing the capacitor value of valve side bushing tap or paralleling more capacitor at the voltage divider both can decrease Xc. However, it is obvious that the capacity of converter transformer bushing tap is hard to change. And paralleling more capacitor at the voltage divider will change second side voltage and induce more other work for protection, measurement and control. For the more, low voltage brings more challenge for the accuracy of AD component.

(2)Increase XL

Redesign the excitation characteristic of the voltage acquisition unit can effectively increase its inductance. More acquisition units connecting in series can also increase the equivalent inductance. However, both method will lead to increase in equipment volume.

The method of increase XL is limited to the characteristics of ferromagnetic material. The saturation point cannot be increased infinitely. The voltage disturbance is extremely great that the voltage acquisition unit is destined to be driven to saturation region. So that, the method of increasing inductance is limited.

(3)Increase the damping

Considering that the $-400 \mathrm{kV}$ disturbance is unavoidable, the effect method to suppress the ferroresonance is to damping the energy in saturated inductor. In the acquisition unit, a resistor of $1 \mathrm{k} \Omega$ is in series. Increase this resistor value probably is most simple method to increase the damping. PSCAD simulation result showed that, when the resistor value is larger than $5 \mathrm{k} \Omega$ ferroresonance will be effectively suppressed. The influence from the increased resistor value on the accuracy of the voltage acquisition unit is shown in figure 10 and figure 11. 


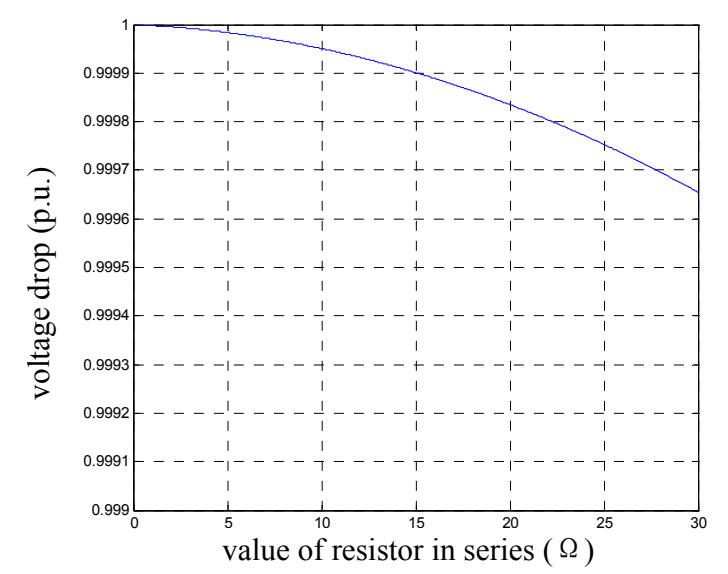

Fig.10 Influence of the resistor value on the accuracy of the voltage acquisition

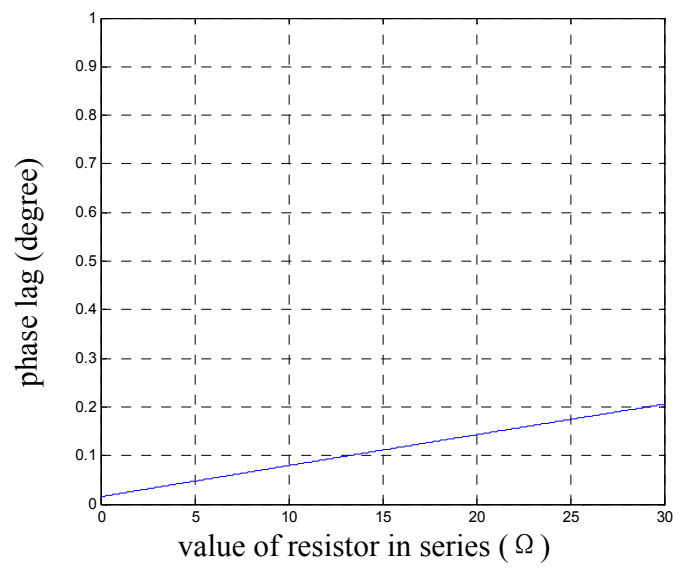

Fig.11 Influence of resistor value on the phase accuracy of voltage acquisition

From the figure above, it can be deduced that the increase of resistor value has little influence on the accuracy of the voltage acquisition unit. The amplitude accuracy could be limited to $0.01 \%$ when the resistor value small than $10 \mathrm{k} \Omega$.

\section{Conclusion}

The charging to connection operation of high-end valve hall in UHV converter station induced a large disturbance to the converter transformers of high-end valve hall, which could easily lead to steady ferroresonance. This paper analyzed the inducement and mechanism of this kind of fault. Simulation models were established to research the transient process of ferroresonance based on PSCAD. Several methods were proposed to suppress the ferroresonance, and theirs characteristic were analyzed. Increase the damping resistor is recommended for its convenience. The influence from the resistor value on the accuracy of the acquisition unit is also analyzed. This method provides a powerful support for the safe and stable operation of UHV converter station.

\section{Reference}

[1] Sanaye-Pasand M, Rezaei-Zare A, Mohseni H, et al. Comparison of performance of various ferroresonance suppressing methods in inductive and capacitive voltage transformers [C]. Power India Conference, New Delhi, India: IEEE, 2006.

[2] Don R Seveik, Charles W Fromen. Experiences with Ferroresonance Problems on EHV Equipment [M]. Alington Texas: Houston Lighting \& Power Compan, 1980.

[3] Rezaei-Zare A, Sanaye-Pasand M, Mohseni H, et al. Analysis of ferroresonance modes in power transformers using Preisach-type hysteretic magnetizing inductance [J]. Power Delivery, 
IEEE Transactions on, 2007, 22(2): 919-929.

[4] Val Escudero M, Dudurych I, Redfern M A. Characterization of ferroresonance modes in HV substation with CB grading capacitors [J]. Electric power systems research, 2007, 77(11):1506-1513.

[5] Mokryani G, Siano P, Piccolo A. Identification of ferroresonance based on S-transform and support vector machine [J]. Simulation Modeling Practice and Theory, 2010, 18(9):1412-1424.

[6] Wornle F, Harrison D K, Zhou C. Analysis of a ferroresonant circuit using bifurcation theory and continuation techniques [J]. Power Delivery, IEEE Transactions on, 2005, 20(1):191-196.

[7] Tran-Quoc T, Pierrat L. An efficient non-linear transformer model and its application to ferroresonance study [J]. Magnetics, IEEE Transactions on, 1995,31(3):2060-2063.

[8] Emin Z, Al Zahawi B A T, Tong Y K, et al. Quantifications of the chaotic behavior of ferroresonant voltage transformer circuits [J]. Circuits and Systems I: Fundamental Theory and Applications IEEE Transactions on, 2001,48(6):757-760.

[9] Huang S J, Hsieh C H. Relation analysis for ferroresonance of bus potential transformer and circuit breaker grading capacitance [J]. International Journal of Electrical Power \& Energy Systems. 2013, 51:61-70.

[10]Abbasi A, Fathi S H, Gharehpatian G B, et al. Voltage transformer ferroresonance analysis using multiple scales method and chaos theory [J]. Complexity, 2013, 18(6):34-45.

[11] Moses P S, Masoum M A S, Toliyat H A. Impacts of hysteresis and magnetic couplings on the stability domain of ferroresonance in asymmetric three-phase three-leg transformers [J]. Energy Conversion, IEEE Transactions on, 2011,26(2):581-592. 